\title{
DISSOCIATIVE SHOCKS IN THE NEIGHBORHOOD OF ORION IRc2 TRACED WITH ATOMIC CARBON
}

\author{
Juan R. Pardo and José Cernicharo \\ Departamento de Astrofísica Molecular e Infrarroja, Instituto de Estructura de la Materia, CSIC, Serrano 121, E-28006 Madrid, Spain; \\ pardo@damir.iem.csic.es, cerni@damir.iem.csic \\ AND \\ Thomas G. Phillips \\ Division of Physics, Mathematics, and Astronomy, California Institute of Technology, Mail Stop 320-47, Pasadena, CA 91125; phillips@ submm.caltech.edu \\ Received 2005 July 19; accepted 2005 October 11; published 2005 November 3
}

\begin{abstract}
We present Caltech Submillimeter Observatory $10^{\prime \prime}$ resolution maps of the ${ }^{3} P_{2} \rightarrow{ }^{3} P_{1}$ line of neutral atomic carbon $(809.3435 \mathrm{GHz})$ and the $J=7-6$ line of $\mathrm{CO}(806.6518 \mathrm{GHz})$ covering a region of $2^{\prime} \times 4^{\prime} .5$ around Orion IRc2 in the BN/KL Nebula. The two lines were observed with the same receiver simultaneously, and therefore the two maps have zero relative pointing error. The atomic carbon $\left(\mathrm{C}_{\mathrm{I}}\right)$ emission does not peak toward the IRc2 position and displays a very different spatial distribution with respect to most molecular species. Moderately strong red wings are detected in $\mathrm{C}_{\mathrm{I}}$ around the region defined by the high-velocity $\mathrm{CO}$ gas with a spatial distribution that delineates a shell of $\sim 20^{\prime \prime}$ radius. We propose that CO dissociation in the J-type shocks produced by the interaction of the IRc2 outflows with the surrounding quiescent gas could play a significant role in this enhancement of atomic carbon in that shell.
\end{abstract}

Subject headings: ISM: individual (Orion IRc2) — ISM: molecules — line: profiles — radio lines: ISM — submillimeter

\section{INTRODUCTION}

Atomic carbon is an important coolant of the neutral interstellar medium (Keene 1995), plays a key role in interstellar chemistry, and is an important tool for probing intermediate regions between diffuse interstellar matter and dense molecular clouds. The first detection of its $492 \mathrm{GHz}^{3} P_{1} \rightarrow{ }^{3} P_{0}$ fine-structure transition was obtained by the Kuiper Airborne Observatory (KAO; Phillips et al. 1980). The ${ }^{3} P_{2} \rightarrow{ }^{3} P_{1}$ transition $(809 \mathrm{GHz})$ was first detected with the University of Hawaii 88 inch $(2 \mathrm{~m})$ telescope (Jaffe et al. 1985). Both lines were first discovered in the Orion molecular cloud. Data from the Cosmic Background Explorer (Wright et al. 1991) have shown that C I emission is widely seen throughout our Galaxy and is generally bright in the Galactic plane. The $\mathrm{C} \mathrm{I} / \mathrm{CO}$ abundance ratio has been confirmed to be high (0.1-1) in nearby galaxies (Stutzki et al. 1997; Gérin \& Phillips 2000). Its detailed distribution in individual Galactic clouds has become a subject of study in the past years, and the rapid gain in submillimeter receiver sensitivity has allowed great progress from both ground-based and satellite platforms.

In the chemically rich and complex environment of the Orion massive-star formation region, $\mathrm{C}$ I is expected to be a good choice to trace the dissociation of the abundant $\mathrm{CO}$ molecule and its possible association with shocks. Phillips \& Huggins (1981) had noticed the trend for atomic carbon not to be as strongly peaked at cloud cores compared with molecules and particularly CO. White \& Padman (1991) pointed out important differences between the spatial distributions of both species in the Orion region and, in particular, that $\mathrm{C}_{\mathrm{I}}$ lies mostly in a shell-like structure around IRc2. The first CO $J=7-6$ (the highest frequency line of $\mathrm{CO}$ that is regularly observed from ground-based observatories) map around Orion IRc2 was obtained with the KAO at 98" resolution (Schmid-Burgk et al. 1989). Recent observations with $\sim 10 \mathrm{~m}$ dishes have improved the angular resolution to $\sim 15^{\prime \prime}$ (Wilson et al. 2001). Large areas of the integral-shaped region of Orion have been recently mapped in the two lowest fine-structure lines of C I (Plume et al. 2000; Yamamoto et al. 2001; Ikeda et al. 2002), but with modest angular resolutions: 1 '.4-2'.2. The region around IRc2 was mapped at the James Clerk Maxwell Telescope using the ${ }^{3} P_{1} \rightarrow{ }^{3} P_{0}$ line $(492 \mathrm{GHz})$ with an angular resolution of 14 " by White \& Sandell (1995).

In this work we present the spatial distribution of atomic carbon and carbon monoxide in a region of $2^{\prime} \times 4{ }^{\prime} .5$ centered on Orion IRc2 using the Caltech Submillimeter Observatory (CSO). Our choice for tracing $\mathrm{CO}$ is through its $J=7-6$ line originating at an energy level of $156 \mathrm{~K}$, which should therefore be especially well adapted for tracing the moderate- and highvelocity "plateau" structure. A C I transition $\left({ }^{3} P_{2} \rightarrow{ }^{3} P_{1}\right)$ is very close in frequency $(809 \mathrm{GHz})$, so these two lines are both accessible with similar atmospheric transmission and can be mapped simultaneously at virtually the same resolution. The observations and results are presented in $\S 2$. The data are discussed in $\S 3$.

\section{OBSERVATIONS AND RESULTS}

The observations were performed with the $10.4 \mathrm{~m}$ telescope of the CSO at the summit of Mauna Kea (Hawaii) in 2000 September. The receiver was a helium-cooled SIS mixer operating in double-sideband mode. For details about the receiver, see Kooi et al. (2000). Two acousto-optic spectrometers with 0.5 and $1.0 \mathrm{GHz}$ coverage providing velocity resolutions of 0.174 and $0.55 \mathrm{~km} \mathrm{~s}^{-1}$, respectively, were used simultaneously during the observations. The pointing was checked every hour using Saturn and was determined to be good within $3^{\prime \prime}$, for a half-power beamwidth of $\sim 10^{\prime \prime}$ at $806 \mathrm{GHz}$. As both lines were observed with the same receiver tuning, relative pointing problems are eliminated.

Atmospheric precipitable water vapor was in the range of $0.7-1.0 \mathrm{~mm}$. The system temperature was $\sim 4000 \mathrm{~K}$. The observations were performed using the on-the-fly procedure with the antenna moving at an angular speed of $1^{\prime \prime} \mathrm{s}^{-1}$ across a row made of 11 boxes of $10^{\prime \prime} \times 10^{\prime \prime}$ each. A calibration scan and an off-position scan $120 \mathrm{~s}$ long, a degree away from IRc2 in 
azimuth, were performed at the end of each row. A total of 297 positions were observed with this procedure.

The calibration is achieved by assuming that the effective temperature of the atmosphere equals the ambient temperature. The data have been corrected for the difference between these temperatures in order to obtain accurate antenna temperatures, $T_{A}^{*}$ (see Pardo et al. 2005). At $806 \mathrm{GHz}$ the correction factor is as high as $15 \%$ at the elevation of the source for $\tau_{\text {atm, zenith }} \sim 1.5$.

Figure 1 shows $\mathrm{CO} J=7-6$ and $\mathrm{C}_{\mathrm{I}}{ }^{3} P_{2} \rightarrow{ }^{3} P_{1}$ spectra at selected positions. Our $\mathrm{CO} 7-6$ data have much better signalto-noise ratios than previous observations obtained at similar angular resolutions due to system temperatures being in our case at least a factor of 4 lower and similar integration times. Toward the position of IRc2 we can clearly see that there is emission between about -90 and $80 \mathrm{~km} \mathrm{~s}^{-1}$. The shape of our signal toward this position coincides quite well with the $\mathrm{CO}$ $J=4-3$ observation of Wilson et al. (2001). The velocity extent of the signal quickly decreases as the distance to IRc2 increases; $1^{\prime}$ away from IRc2 the line is already less than $30 \mathrm{~km} \mathrm{~s}^{-1}$ wide. Only the extended ridge is then detected. $\mathrm{CO}$ and $\mathrm{C} \mathrm{I}$ emission also displays line wings at position $\Delta \alpha=-10^{\prime \prime}, \Delta \delta=-80^{\prime \prime}$, i.e., in the direction of the Orion cross-outflow (Schmid-Burgk et al. 1990).

Our $\mathrm{C}_{\text {I }}{ }^{3} P_{2} \rightarrow{ }^{3} P_{1}$ data, in addition to having much better angular resolution, also significantly improve the signal-tonoise ratio and the velocity resolution, with respect to those presented in Yamamoto et al. (2001). The Plume et al. (2000) and Ikeda et al. (2002) data of the lower ${ }^{3} P_{1} \rightarrow{ }^{3} P_{0}$ transition have a poor angular resolution, $\simeq 2$ ' in both cases, and comparison with our results is difficult. The White \& Sandell (1995) map of the lower transition is the only one useful for comparison with our C I map (see next section).

\section{DISCUSSION}

The features that have been revealed in the Orion Nebula by the large number of millimeter and submillimeter observations available are the following: First, a dense and warm clump called the "hot core." It is quite compact $\left(10^{\prime \prime}\right)$, with temperatures estimated in the range $400-500 \mathrm{~K}$ and densities reaching $10^{7} \mathrm{~cm}^{-3}$. However, the detection of $\mathrm{CO}$ transitions with upper rotational levels up to $J=43$ reveal the presence of a much hotter component with $T=1500-2000 \mathrm{~K}$ and CO column densities on the order of $2 \times 10^{17} \mathrm{~cm}^{-2}$ (Sempere et al. 2000). Second, a moderate velocity "plateau" consisting of gas heated to temperatures ranging from 80 to $200 \mathrm{~K}$ distributed in a region covering $\sim 40^{\prime \prime}$ centered on IRc2 with an average density below $3 \times 10^{6} \mathrm{~cm}^{-3}$. Lines arising from this component typically have half-power line widths of about $40-50 \mathrm{~km} \mathrm{~s}^{-1}$ (see, for example, HCN and HDO in Pardo et al. 2001), although high-velocity gas is seen in $\mathrm{CO}$ in the velocity ranges -60 to -40 and 50 to $80 \mathrm{~km} \mathrm{~s}^{-1}$. Finally, a much extended structure called the "ridge" with densities of $\sim 10^{4}-10^{5} \mathrm{~cm}^{-3}$, velocity widths of $\sim 8 \mathrm{~km} \mathrm{~s}^{-1}$, and temperatures still reaching $50-60 \mathrm{~K}$ due to heating by the UV field from the Trapezium stars. The emission from and the radiative transfer through all these components together give a quite complex output.

The high-velocity (40-60 $\mathrm{km} \mathrm{s}^{-1}$ ) CO 7-6 emission peaks on IRc2 and also on the $\mathrm{H}_{2}$ peak 2 (see Sugai et al. 1994 as a reference for Orion's molecular hydrogen peaks). The extremely high velocity CO gas $\left( \pm 60-80 \mathrm{~km} \mathrm{~s}^{-1}\right)$ is found along a filament connecting the positions of IRc2 and $\mathrm{H}_{2}$ peak 1 . This filament is not necessarily a jet, but the result of heating

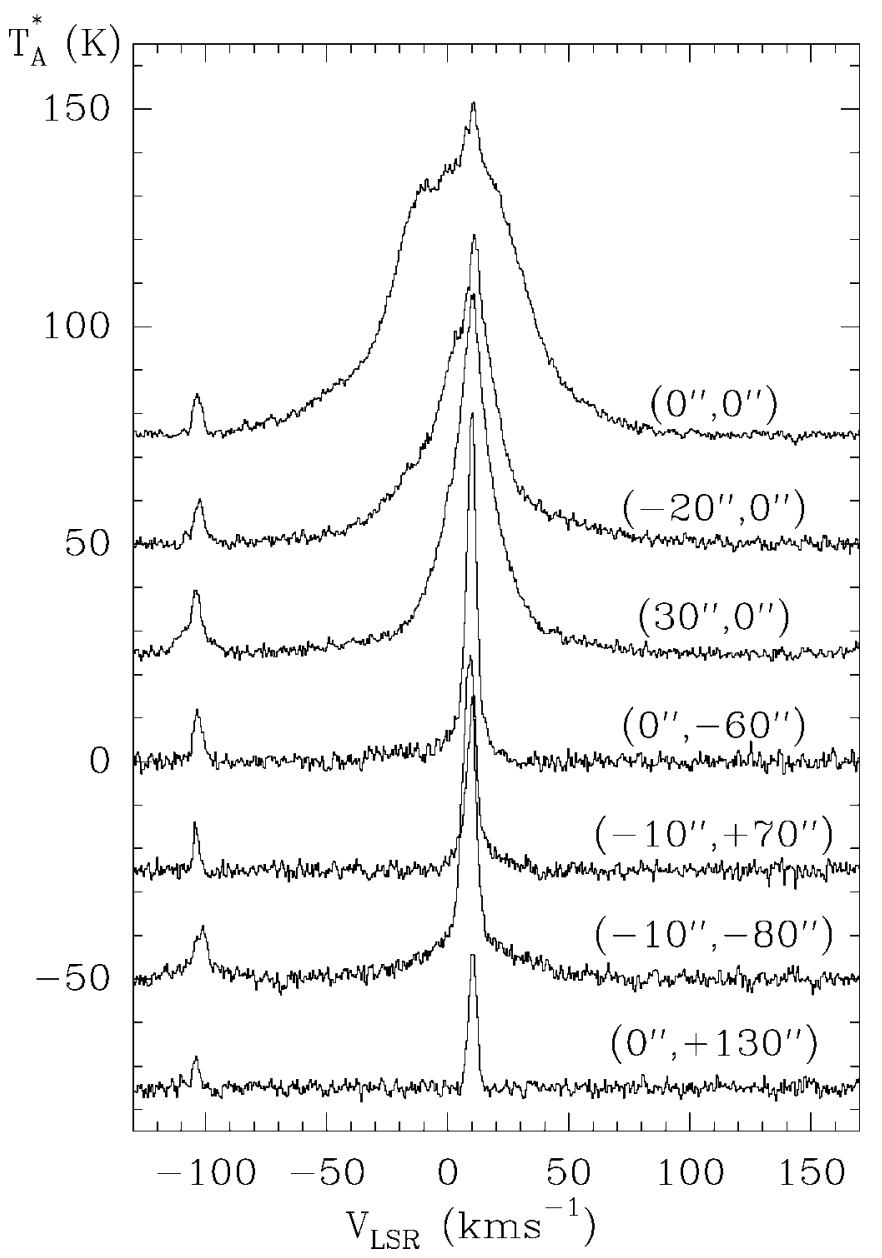

FIG. 1.-CO $J=7-6$ spectra at selected positions around Orion IRc2 $\left(\alpha=5^{\mathrm{h}} 35^{\mathrm{m}} 14.5, \delta=-5^{\circ} 22^{\prime} 31^{\prime \prime}\right.$ [J2000.0]). The line at $-105 \mathrm{~km} \mathrm{~s}^{-1}$ is the ${ }^{3} P_{2} \rightarrow{ }^{3} P_{1}$ transition of atomic carbon from the image sideband. Therefore, the velocity scale for it has to be recentered and reversed.

by the embedded sources of the gas inside the cavity eroded by Orion's outflows. Although the filament is mainly seen at extreme velocities, emission at all velocities exists along it. This spatial distribution of the high-excitation $\mathrm{CO}$ emission is rather different from the one traced by its low- $J$ lines. Moreover, the spatial distribution of the extreme negative and positive velocities in the CO $J=7-6$ emission looks very similar to that of water vapor at $183.3 \mathrm{GHz}$, as observed by Cernicharo et al. (1994). Taking into account that this line of water is extremely sensitive to the kinetic temperature, its spatial coincidence with the CO 7-6 line reinforces the idea that the latter is tracing a warm-gas component, probably related to the one traced by the high-excitation lines of CO (up to $J=43$ ) observed with the Infrared Space Observatory by Sempere et al. (2000) and the rovibrational lines of $\mathrm{CO}$ and $\mathrm{H}_{2} \mathrm{O}$ observed by González-Alfonso et al. (2002).

The spatial distribution of $\mathrm{C}$ I obtained in this work provides a clearer picture than previously. Toward IRc2 the line peaks at $\sim 9-10 \mathrm{~km} \mathrm{~s}^{-1}$ and has a width at half-intensity on the order of $5 \mathrm{~km} \mathrm{~s}^{-1}$. However, at other positions (for example, see position $\Delta \alpha=30^{\prime \prime}, \Delta \delta=0^{\prime \prime}$ in Fig. 1) moderate wings are clearly detected and an interesting velocity structure is revealed (see Fig. 2). The integrated line intensity delineates a doublepeaked structure with the two peaks defining a line quite close to that of the central section of the integral-shaped large-scale structure of the Orion Nebula. The highest $\mathrm{C}_{\mathrm{I}}{ }^{3} P_{2} \rightarrow{ }^{3} P_{1}$ intensity 

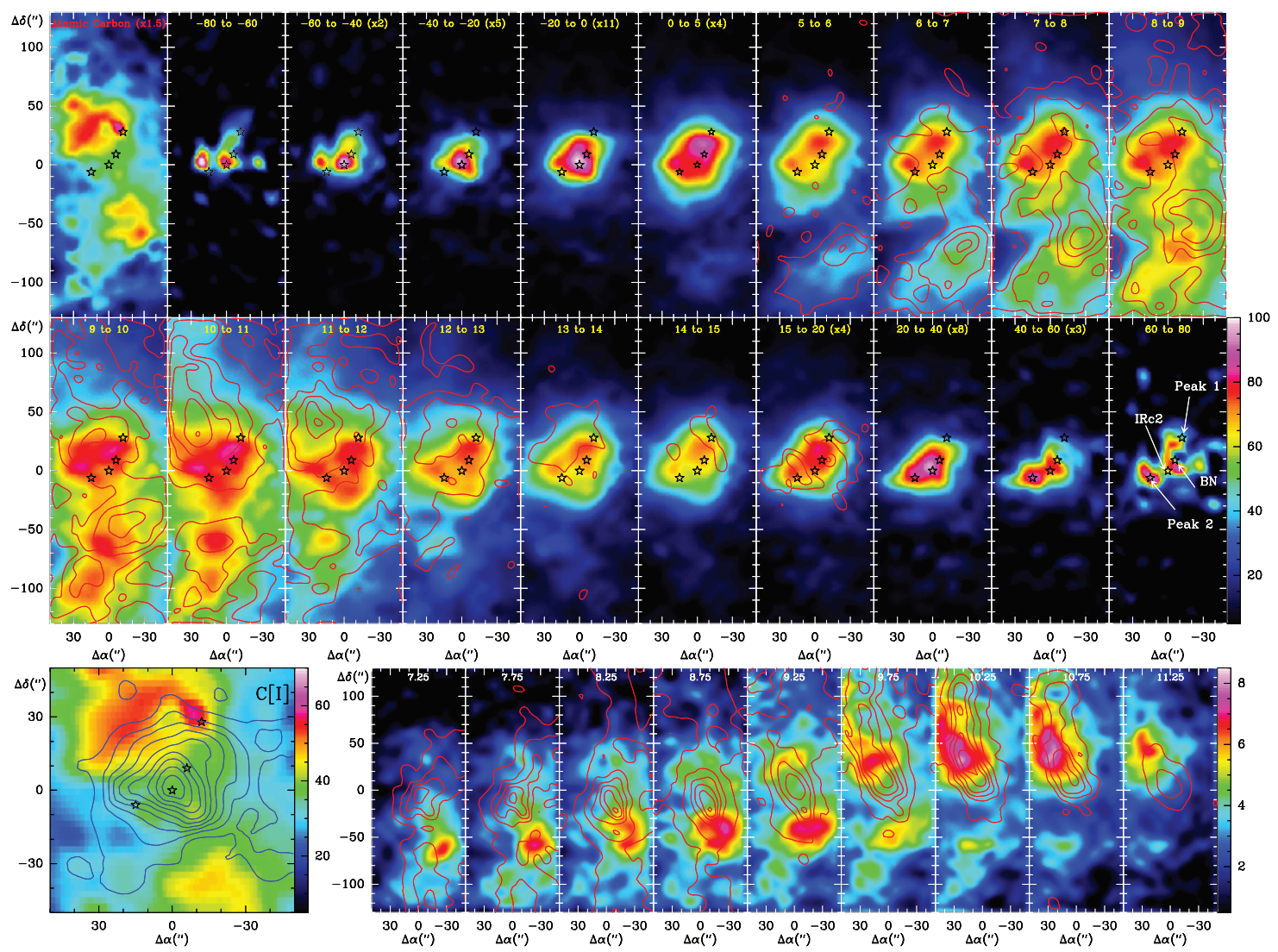

Fig. 2.-Top two rows: Color map at the top left shows the observed total integrated line intensity of the ${ }^{3} P_{2} \rightarrow{ }^{3} P_{1}$ transition of atomic carbon. The other panels of the top two rows show the $\mathrm{CO} J=7-6$ integrated intensity at different velocity ranges (indicated at the top of each panel). The integrated intensity for some maps has been divided by a scale factor (indicated in the panels as necessary) to maintain the same color scale for all maps (the units for all color maps in this figure are $\mathrm{K} \mathrm{km} \mathrm{s}^{-1}$ ). The panels corresponding to velocities from 0 to $20 \mathrm{~km} \mathrm{~s}^{-1}$ also show as red contours the integrated intensity in the corresponding interval of atomic carbon (first contour and step are both $2 \mathrm{~K} \mathrm{~km} \mathrm{~s}^{-1}$ ). The positions of BN, IRc2, and $\mathrm{H}_{2}$ peaks 1 and 2 (see text) are marked for reference and identified in the $60-80 \mathrm{~km} \mathrm{~s}^{-1}$ map. Bottom row: The left panel is a zoom of the integrated intensity map of atomic carbon over the central region of Orion IRc2. The contours correspond to the blue (from -40 to $-20 \mathrm{~km} \mathrm{~s}^{-1}$ ) high-velocity $\mathrm{CO} J=7-6$ emission. Note that the atomic carbon integrated intensity has its maximum at the edge of the region where the high-velocity $\mathrm{CO}$ gas is present. The right panels show the atomic carbon intensity at different velocities (indicated in each panel) over a range of $0.5 \mathrm{~km} \mathrm{~s}^{-1}$. The contours correspond to the emission of the $J=12-11$ transition of $\mathrm{HC}_{3} \mathrm{~N}$ as observed with the $30 \mathrm{~m}$ IRAM telescope and kindly provided by A. Rodríguez-Franco (see Rodríguez-Franco et al. 1992; first contour and step are both $0.5 \mathrm{~K} \mathrm{~km} \mathrm{~s}^{-1}$ ).

is found virtually in coincidence with the shocked molecular hydrogen peak 1 . The southwest $\mathrm{C}$ i peak is mainly blueshifted emission, whereas the northeast, more intense peak is redshifted with respect to the peak velocity of $\mathrm{CO}, \sim 9 \mathrm{~km} \mathrm{~s}^{-1}$. The ${ }^{3} P_{1} \rightarrow{ }^{3} P_{0}(492 \mathrm{GHz})$ maps presented in White \& Sandell (1995) also have two peaks, northeast and southwest of IRc2, although slightly more separated than ours. The most prominent peak in their case is also the northeast one, which has the shape of an arc, relatively similar to what we see. The velocity-binned maps of ${ }^{3} P_{1} \rightarrow{ }^{3} P_{0}$ suggest that $\mathrm{C}$ I delineates a shell-like structure around IRc2, a result that is now much better supported by our velocity-binned maps of ${ }^{3} P_{2} \rightarrow{ }^{3} P_{1}$ presented in Figure 2 . The $\mathrm{C}$ I contours for the $15-20 \mathrm{~km} \mathrm{~s}^{-1}$ velocity range still seem to provide a hint of Orion's outflow.

In our case, especially for the northeast $\mathrm{C}$ I peak between 10 and $12 \mathrm{~km} \mathrm{~s}^{-1}$ but also for the southwest one, a puzzle-like complementarity with the high-velocity (both wings) $\mathrm{CO}$ $J=7-6$ emission suggests that shocks created by the interaction of the central outflow with the extended gas component dissociate $\mathrm{CO}$ and therefore cause the enhancement of atomic carbon in the shock fronts. The radius of the delineated C I shell is $\sim 20^{\prime \prime}$. Combined with this, in the large-scale distribution of atomic carbon other factors should play a role, such as intrinsic differences in column density and temperature and the effect of UV photons from the nearby ionizing $\mathrm{O}$ and B stars.

In order to check the effect that volume density differences may have on the observed C I spatial distribution, it is helpful to compare with a high-density tracer. The $\mathrm{HC}_{3} \mathrm{~N} J=12-11$ data from Rodríguez-Franco et al. (1992) have been kindly provided by the authors. The comparison of $\mathrm{C}$ I and $\mathrm{HC}_{3} \mathrm{~N}$ $J=12-11$ spatial distributions at different velocities is also shown on Figure 2. For the velocity range $7-9 \mathrm{~km} \mathrm{~s}^{-1}$, the $\mathrm{HC}_{3} \mathrm{~N}$ emission is well centered on IRc2, but when the northeast C I peak appears at $v>9 \mathrm{~km} \mathrm{~s}^{-1}$, the $\mathrm{HC}_{3} \mathrm{~N}$ peak moves to be almost coincident with it, at least in the range $9.5-11.0 \mathrm{~km} \mathrm{~s}^{-1}$. This fact indicates that the stronger intensity of the northeast C I peak with respect to the southwest one is probably due to a larger gas column density there.

The column density of atomic carbon can be estimated from its two fine-structure lines. The data from White \& Sandell (1995) for the ${ }^{3} P_{1} \rightarrow{ }^{3} P_{0}$ C I line can be compared with ours for ${ }^{3} P_{2} \rightarrow{ }^{3} P_{1}$ with a similar spatial resolution. As discussed by these authors, the excitation temperatures of the atomic carbon lines are not very sensitive to the physical parameters of the different gas components of the Orion cloud. One could assume that the atomic carbon lines are thermalized with an excitation tem- 
perature close to that of the carbon monoxide lines. The observed intensities for both lines suggest excitation temperatures close to $100 \mathrm{~K}$. We obtain column densities for $\mathrm{C}_{\mathrm{I}}$ in the range $(0.2-2) \times 10^{18} \mathrm{~cm}^{-2}$ using the equation proposed by Keene et al. (1985), but correcting for the line strength ratio between the ${ }^{3} P_{2} \rightarrow{ }^{3} P_{1}$ and ${ }^{3} P_{1} \rightarrow{ }^{3} P_{0}$ lines. The deduced abundance ratio between atomic carbon and carbon monoxide varies therefore between $\sim 0.01$ and 0.35 according to the results found for $\mathrm{CO}$ by White \& Sandell (1995) using $\mathrm{C}^{18} \mathrm{O}$ and ${ }^{13} \mathrm{CO}$, and our own calculations using an LVG code with $T_{K}=100 \mathrm{~K}$ (CO column density in the range $6 \times 10^{18}$ to $3 \times 10^{19}$ ). It is reasonable to think that the maximum is located at the shell of enhanced $\mathrm{C}_{\mathrm{I}}$ emission and that the minimum is located in the direction of IRc2, although new detailed observations of optically thin lines of isotopic $\mathrm{CO}$ at similar resolutions would be necessary to confirm this point.

The fact that $\mathrm{C}_{\mathrm{I}}$ is the result of the dissociation, driven by photons or shocks, of a molecule with a large abundance in molecular clouds (CO) and having lines in the submillimeterwave region, where appropriate resolutions can be achieved with available telescopes, has provided this unique opportunity to trace this dissociation in the vicinity of Orion IRc2. Other atomic species (i.e., O I with SOFIA and Herschel) and radicals should be mapped at similar or higher resolutions in order to check whether or not the effect of dissociative shocks in the neighborhood of Orion IRc2 is the main thing responsible for what has been presented in this letter.

\section{SUMMARY}

CO 7-6 and $\mathrm{C}$ I ${ }^{3} P_{2} \rightarrow{ }^{3} P_{1}$ mapping around Orion IRc2 with high angular resolution $\left(\sim 10^{\prime \prime}\right)$ has been achieved with no relative pointing problems. The data reveal a double-peaked structure for the distribution of $\mathrm{C}_{\mathrm{I}}$ in the neighborhood of Orion IRc2, indicating a shell of radius $\sim 20^{\prime \prime}$, that shows a striking complementarity with the distribution of high-velocity $\mathrm{CO} 7-6$. It is proposed that this structure could be evidence of a shockdriven dissociation of $\mathrm{CO}$ produced as the molecular outflow impinges on the surrounding gas. Local differences in the general column density have been explored using a high-density tracer $\left(\mathrm{HC}_{3} \mathrm{~N}\right)$, which seems to explain why one of the $\mathrm{C} I$ peaks (to the northeast) is stronger.

This work has been supported by Spanish DGES and PNIE grants AYA2000-1784, ESP2001-4516, and AYA2003-2785 and by US NSF grant AST 99-80846.

\section{REFERENCES}

Cernicharo, J., González-Alfonso, E., Alcolea, J., Bachiller, R., \& John, D. 1994, ApJ, 432, L59

Gérin, M., \& Phillips, T. G. 2000, ApJ, 537, 644

González-Alfonso, E., Wright, C. M., Cernicharo, J., Rosenthal, D., Boonman, A. M. S., \& van Dishoeck, E. F. 2002, A\&A, 386, 1074

Ikeda, M., Oka, T., Tatematsu, K., Sekimoto, Y., \& Yamamoto, S. 2002, ApJS, 139,467

Jaffe, D. T., Harris, A. I., Silber, M., Genzel, R., \& Betz, A. L. 1985, ApJ, 290, L59

Keene, J. 1995, in The Physics and Chemistry of Interstellar Molecular Clouds, ed. G. Winnewisser \& G. Pelz (Berlin: Springer), 186

Keene, J., Blake, G. A., Phillips, T. G., Huggins, P. J., \& Beichman, C. A. 1985, ApJ, 299, 967

Kooi, J. W., et al. 2000, Int. J. Infrared Millimeter Waves, 21, 1357

Pardo, J. R., Cernicharo, J., Herpin, F., Kawamura, J., Kooi, J., \& Phillips, T. G. 2001, ApJ, 562, 799

Pardo, J. R., Serabyn, E., \& Wiedner, M. 2005, Icarus, 178, 19

Phillips, T. G., \& Huggins, P. J. 1981, ApJ, 251, 533
Phillips, T. G., Huggins, P. J., Kuiper, T. B. H., \& Miller, R. E. 1980, ApJ, 238, L103

Plume, R., et al. 2000, ApJ, 539, L133

Rodríguez-Franco, A., Martín-Pintado, J., Gómez-González, J., \& Planesas, P. 1992, A\&A, 264, 592

Schmid-Burgk, J., Güsten, R., Mauersberger, R., \& Schulz, A. 1990, ApJ, 362, L25

Schmid-Burgk, J., et al. 1989, A\&A, 215, 150

Sempere, M. J., Cernicharo, J., Le Floc'h, B., González-Alfonso, E., \& Leeks, S. 2000, ApJ, 530, L123

Stutzki, J., et al. 1997, ApJ, 477, L33

Sugai, H., et al. 1994, ApJ, 420, 746

White, G. J., \& Padman, R. 1991, Nature, 354, 511

White, G. J., \& Sandell, G. 1995, A\&A, 299, 179

Wilson, T. L., Mudders, D., Kramer, C., \& Henkel, C. 2001, ApJ, 557, 240

Wright, E. L., et al. 1991, ApJ, 381, 200

Yamamoto, S., et al. 2001, ApJ, 547, L165 